\title{
Der Schutz der subjektiven Rechte in der kolumbianischen Verfassung ${ }^{1}$
}

\author{
Von Andreas Timmermann
}

\section{Einführung}

Die seit der Conquista gewachsenen Machtstrukturen in Lateinamerika haben bis in die Gegenwart einen nicht zu unterschätzenden Einfluß auf die Ausgestaltung der Verfassungsgarantien. Hinzu kommt das Vorbild des europäischen Konstitutionalismus, das dazu beigetragen hat, die Freiheits- und Abwehrrechte vielfach stark zu betonen ${ }^{2}$. Dennoch fanden als Erwiderung auf die nahezu absoluten, bis in das 20.Jahrhundert hinein quasi feudalistischen Befugnisse der Oberschicht frühzeitig auch soziale Grundrechte als "Prototyp der Teilhaberechte" ${ }^{3}$ Aufnahme in einzelne Verfassungsurkunden. Maßgeblich waren zumeist reformsozialistische Bestrebungen; so im Fall der mexikanischen Verfassung von $1917^{4}$. Teilweise wurden sie zusätzlich befördert durch die katholische Soziallehre, wie das Beispiel Costa Ricas belegt. ${ }^{5}$

Vor diesem Hintergrund verdient auch das kolumbianische Beispiel Beachtung und eine eigene Würdigung. Seit den ersten verfassungberatenden Versammlungen noch während des Unabhängigkeitskrieges mit der Spanischen Krone stehen die Garantien von Freiheit und Eigentum neben den Forderungen nach Gleicheit und Ablösung der Standesprivile-

1 Überarbeitete Fassung eines Vortrags vor dem Arbeitskreis "Überseeische Verfassungsvergleichung" am 27.6.1998. in: Hans-Rudolf Horn/Albrecht Weber (Hg.): Richterliche Verfassungskontrolle in Lateinamerika, Spanien und Portugal, Baden-Baden 1989, S. 105 (108).

3 Peter Badura: Staatsrecht, München 1986, S. 63.

4 Vgl. Art. 3 (Recht auf Arbeit), Art. 27 (Agrarreform) und Art. 123 im 6.Titel ("Arbeit und soziale Sicherung"), näher Hans-Rudolf Horn: Grundzüge des mexikanischen Verfassungsrechts, in: Jahrbuch des öf fentlichen Rechts (JöR) N.F. 29 (1980), S. 479 (492ff).

Ansätze einer sozialen Verbürgung finden sich in der Verfassung von 1917, ausgeprägt in der Verfassung von 1949, Jochen Fuchs: Die Verfassung der Republik Costa Rica, in JöR N.F. 35 (1986), S. 425 (443ff); ders.: Costa Rica und seine Verfassung vom 7. November 1949 in Vergangenheit und Gegenwart, in: VRÜ 20 (1987). S. 177 (185f). 
gien $^{6}$. Bedeutsame Etappen sind die Verfassung von 1832 mit der Aufhebung des gutsherrlichen Ältestenrechts (Majorat) und die Verfassung von 1853 mit dem Verbot der Sklave$\mathrm{rei}^{7}$. Allerdings legitimierten die ihrem Ursprung nach liberalen, aufklärerischen Grundrechtserklärungen im Ergebnis die bestehenden feudalen Besitz- und Herrschaftsverhältnisse. Die rechtlich und faktisch unbeschränkte wirtschaftliche Freiheit hat die beachtliche Machtkonzentration auf dem Lande und die Bildung wirtschaftlicher Monopole noch im zwanzigsten Jahrhundert begünstigt.

Erst die Reform der kolumbianischen Konstitution von 1886 im Jahre 1936 rückte die mit der Wirtschaftsfreiheit verbundene soziale Frage in den Mittelpunkt der Verfassungsdebatte. Wichtige Bestandteile der Reform waren die Erweiterung des Grundrechtekatalogs um soziale Rechte, wie das Recht auf Arbeit, verbunden mit der Pflicht hierzu, und die Aufnahme einer Sozialbindungsklausel in den Eigentumsartikel. Hinzu traten der Auftrag an den Staat, das Ziel der Vollbeschäftigung zu verfolgen, sowie die Ermächtigung des Staates zur Wirtschaftslenkung und -planung ${ }^{8}$. Maßgeblich war nicht nur das Vorbild der mexikanischen Sozialverfassung sowie der Verfassungen von Weimar (1919) und der spanischen Republik (1931). Einen bedeutenden Einfluß hatte ferner die zu Beginn des 20. Jahrhunderts äußerst schwierige Lage der Landbevölkerung, die sich mit der einsetzenden Landflucht bald auch in den rasant wachsenden städtischen Ballungszentren verschlechterte. In dem Maße, in dem die gesellschaftliche Frage in den Vordergrund rückte, gewannen nun auch die öffentlichen Gewalten an Bedeutung, die zunehmend in die Wirtschafts- und Sozialordnung eingriffen. Zugleich relativierte sich das herkömmliche Verständnis des Staates als Wächter über unveräußerliche individuelle Rechte ${ }^{9}$. Folgerichtig nahm etwa die Änderung der Eigentumsordnung eine Schlüsselstellung ein, sowohl aus Sicht der Reformer des Jahres $1936^{10}$ als auch nach Auffassung des Gesetzgebers der

6

7

10

Näher Andreas Timmermann: Soziale Funktion und Umweltfunktion des Eigentums in der spanischen und in der kolumbianischen Verfassung, Baden-Baden 1997, S. 140f.

Alvaro Tirado Mejía: Propiedad, intervención y planeación en las Constituciones colombianas, in: Economía Colombiana Nr.234 (1991), S. 12 (13f).

Näher Jesús Vallejo Mejía: El Estado Social de Derecho en Colombia, in: Alvaro Tirado Mejía (Hg.): Estado y Economía. 50 años de la reforma de 1936, Bogotá 1986, S. 113 (117ff); Guillermo Chaín Lizcano: La cuestión económica en el constitutionalismo colombiano, in: Universidad Nacional de Colombia (Hg.): Constitución política y reorganización del Estado, Bogotá 1994, S. 71 (89ff); speziell zu den Reformen im Bereich Beschäftigung Pedro Pacauchique Avila: El carácter internacional del Derecho del Trabajo, su universalidad y alcance en la nueva Constitución colombiana, in: Externado 1991, Nr. 2, S. 17 (24f).

Alfredo Vázquez Carrizosa: La reforma constitucional de 1936 y la intervención del estado en la economía, in: Tirado Mejía (Fn. 8), S. 25 (40f); Vallejo Mejía (Fn. 8), S. 113 (119f); Chaín Lizcano (Fn. 8), S. 71 (88f).

Verfassungskommission des Repräsentantenhauses in: Anales de la Cámera de Representantes -(13.03.1936) 2.Serie, Nr.189. 
kommenden Jahrzehnte ${ }^{11}$. Denn die äußerst ungleiche Vermögensverteilung seit der spanischen Conquista wird bis heute als einer der Hauptgründe für viele soziale Verwerfungen in Kolumbien angesehen. ${ }^{12}$

\section{Die Anliegen der Konstituante}

Die neue Verfassung von $1991^{13}$ beantwortet "trotz mancher Verfassungslyrik" ${ }^{14}$ die soziale Frage sehr entschieden und mit einer eigenständigen Konzeption der Individualrechte. Diese Konzeption ist nur begrenzt auf westeuropäische Vorbilder zurückzuführen; so auf die allgemeinen Grundrechtslehren im Anschluß an die spanische Verfassung von 1978 beziehungsweise an das Bonner Grundgesetz. Hinzu kommen lateinamerikanische Traditionslinien, die beispielsweise im Gerichtsschutz zu erkennen sind. Während der über hundertjährigen Geltung der Verfassung von 1886 konnte sich eine spezifisch kolumbianische, im ganzen recht pragmatische Verfassungslehre entwickeln, die durch ihre Fortentwicklung in Rechtsprechung und Literatur ein beachtliches Maß an Akzeptanz und Kontinuität erlangt hat ${ }^{15}$. Die kolumbianische Konstituante des Jahres 1991 war bemüht, diesen Zusammenhang zu wahren. Zugleich mußte sie sich den gesellschafts- und wirtschaftspolitischen Erfahrungen sowie grundsätzlichen Erwägungen stellen. Dies betrifft die Allmacht neben- und außerstaatlicher Protagonisten (Milizen, Mafia, Guerilla) sowie die trotz beinahe zweihundertjähriger republikanischer Tradition unzulängliche politische Teilnahme der Bevölkerung und deren geringe Identifikation mit dem Staat. Hinzu kommen institutionelle Schwächen und die mangelnde Rechtsdurchsetzung, gefördert durch einseitige Deutungen der Wirtschafts- und Sozialverfassung im Verlaufe ihres Interpretations- und Konkretisierungsprozesses. Das Ergebnis ist ein vielschichtiger Ansatz, der zwischen den

11 Verwiesen sei auf die kolumbianischen Agrarreformgesetze, ausgehend von der Ley 200/36 vom 30.12.1936 ("reforma agraria"), in: Diario Oficial (DO) Nr.23388 (21.01.1937), über die Ley 135/61 vom 13.12.1961 ("de la reforma social agraria"), in: DO Nr.30691 (20.12.1961), modifiziert durch die Ley 4/73 vom 29.03.1973, in: DO Nr.33828 (13.04.1973), bis hin zur Ley 160/94 vom 03.08.1994 ("por la cual se crea el Sistema Nacional de Reforma Agraria y Desarrollo Rural Campesino"), in: DO Nr.41479 (5.8.1994); ferner auf die Bemühungen um eine städtische Bodenreform, so mit der Ley 9/1989 vom 11.01 .1989 ("sobre la reforma urbana"), in: DO Nr.38650 (11.01.1989), und der Ley 388/97 vom 18.07.1997 ("por la cual se modifican la ley 9a de 1989, y la ley 39 de 1991 "), in: Derecho Colombiano Nr.429 (1997), S. $205 f f$.

M.w.N. Timmermann (Fn. 6), S. 149ff.

Die Verfassung mit insgesamt 380 Artikeln ist abgedruckt in: Derecho Colombiano Nr.418f (1996), S. 5ff.

Matthias Herdegen: Investitionsschutz in Lateinamerika: Neuere Entwicklungen im Verfassungsund Völkervertragsrecht, in: Zeitschrift für Vergleichende Rechtswissenschaft 94 (1995), S. 341 (342).

15

Julio César Ortiz: El sistema de control constitucional en Colombia, in: Boletín Mexicano de Derecho Comparado 24 (1991), S. 481 (485f). 
Bestrebungen nach Kontinuität und Konsens auf der einen sowie verfassungsrechtlicher Neuerung und Gesellschaftsreform auf der anderen Seite einen Kompromiß finden mußte. Die Verfassungsväter strebten danach, den zahlreichen bisher benachteiligten gesellschaftlichen und ethnischen Gruppen des Landes ein Höchstmaß an politischer und gesellschaftlicher Teilhabe zu gewährleisten ${ }^{16}$. Zugleich sollten die Mindestvoraussetzungen einer menschenwürdigen Existenz - Ernährung, Wohnung, Gesundheit und Bildung - Verfassungsrang erhalten ${ }^{17}$. Nicht nur die Landflucht und die weit verbreitete Armut hatte Einfluß auf die Beratungen der Konstituante. Der Verfassungstext sollte auch aktuellen politischen Fragen gerecht werden, unter anderem dem globalen Umweltschutz sowie dem Guerillaund Drogenkrieg, Erscheinungen, welche in unheilvoller Wechselwirkung die in allen öffentlichen Instanzen anzutreffende Korruption weiter befördert haben.

Folgerichtig wurden mit der Suche nach verfassungsrechtlichen Antworten auf neue Fragen auch neue Institutionen geschaffen, wie das kolumbianische Verfassungsgericht (Art. 239$245 \mathrm{CP}^{18}$ ) und nach skandinavischem Vorbild der Bürgeranwalt (Defensor del Pueblo, Art. 281-284 CP). Diese Einrichtungen sollen, verbunden mit dem gleichfalls neuen Institut des Rechtsbehelfs acción de tutela, quasi auf dem Rechtsweg zur besseren Teilhabe und zu einer "allmählichen Demokratisierung der kolumbianischen Gesellschaft" beitragen ${ }^{19}$. Inhaltlich blieb die Zielsetzung der Konstituante angesichts der auseinanderstrebenden gesellschaftlichen Kräfte ausgleichend und hinsichtlich des Handlungsspielraums der öffentlichen Gewalten durchaus realistisch, insgesamt eher pragmatisch als dogmatisch. Eindeutig ist das Bekenntnis der Verfassung zu einer pluralistischen Gesellschaft, deren

Das Verfassungsgericht erwähnt die Schutzbedürftigkeit von 81 verschiedenen ethnischen Gruppen mit 64 Sprachen in Kolumbien, in ihrer Mehrheit Eingeborenengemeinschaften, Sentencia de la Corte Constitucional (SCC) Nr.T-380/98, in: Relatoría Corte Constitucional (RCC) 1993 Nr.49, S. 15; zum Verfassungsprinzip der ethnischen Vielfalt (Art. 7 CP) und zum Rechtsschutz der Eingeborenen SCC Nr.C-139/96 vom 09.04.1996, in: Foro Colombiano Nr.324 (1996), S. 515 (519ff); ferner SCC Nr.SU-039/97 vom 3.2.1997, in: Foro Colombiano Nr.334 (1997), S. 310 (322ff); und SCC Nr.T-523/97 vom 15.10.1997, in: Derecho Colombiano Nr.432 (1997), S. 509 (514ff).

Diese Vorsätze prägten nahezu alle Debatten der fïnften verfassungsberatenden Kommission über wirtschaftliche, soziale und ökologische Fragen, so in den Beratungen vom 17.04. bis 19.04.1991, in: Informe de sesiones de la comisión quinta del día 17 de abril de 1991 und folgende; vgl. ferner die Begründung zum ersten Verfassungsentwurf der Kommission, in: Gaceta Constitucional Nr.46 15.04.1991.

19

So der Präsident des kolumbianischen Verfassungsgerichts mit einer positiven Bilanz zum fünften Jahrestag der Verfassung am 04.07.1996. Von 1991 bis Ende Juni 1996 waren bereits 102.130 Tutela-Anträge beim Verfassungsgericht eingegangen; in: El Espectador (Bogotá) vom 30.06.1996, S. 5A 
Grundlage die Garantien des sozialen Rechtsstaates sind (Art. $1 \mathrm{CP})^{20}$. Die sozialpolitischen Forderungen beantwortete die ganz überwiegende Zahl der Verfassungsväter nicht etwa mit einem Plädoyer für eine sozialistische Gesellschaftsreform oder für eine umfassende Verstaatlichung der Produktionsmittel. Vielmehr plädierten sie für eine gerechtere Verteilung des Einkommens und für einen besseren Zugang zu den vorhandenen materiellen und immateriellen Gütern ${ }^{21}$. Ganz in diesem Sinne betont auch das Verfassungsgericht zunächst die Garantie der Grundrechte und die Verwirklichung der wirtschaftlichen Freiheiten. Andererseits stellt es die Gewährleistungen unter den Vorbehalt einer tatsächlichen, wirksamen Korrektur der bestehenden Ungleichheit und Ungerechtigkeit in der kolumbianischen Gesellschaft; unter diesem Gesichtspunkt seien in der Verfassung die Eingriffsbefugnisse der öffentlichen Gewalten gestärkt worden. ${ }^{22}$

\section{Merkmale des Rechtsschutzes}

Vor diesem Hintergrund sind die Merkmale einer kolumbianischen Lehre von den subjektiven Verfassungsrechten zu erklären. Diese erscheint aus europäischer Sicht teilweise ungewöhnlich, manchmal auch widersprüchlich. Jedoch zeigt sich auf den zweiten Blick in den verschiedenen Rechtsfiguren und in den wiederkehrenden Interpretationsmustern eine innere Konsequenz, welche durch die Prärogative der sozialpolitischen Anliegen geprägt ist.

\section{a. Die Systematisierung}

Kennzeichnend für den Rahmen des subjektiven Rechtsschutzes ist eine Neigung zur Systematisierung, die mit einer besonderen Betonung der Gliederung und Klassifizierung in der gesamten Verfassungsordnung einhergeht. Diese ist nach der Interpretation des Verfassungsgerichts in vier grundlegende Ordnungen des gesellschaftlichen Lebens eingeteilt, nämlich in eine jeweils eigenständige Wirtschafts-, Sozial-, Kultur- und Umweltverfas-

20

Diesbezüglich anerkennend aus europäischer und rechtsvergleichender Sicht Matthias Herdegen: El desarrollo constitucional colombiano a la luz del derecho comparado, in: Univérsitas Nr.86 (1994), S. 7 (8ff).

21 Dazu Alberto Toro Lopera: Principios fundamentales de la Constitución Política de la República de Colombia, 2.Auflage, Medellín 1993, S. 168f; Pedro Antonio Lamprea Rodríguez: Principios fundamentales en la Constitución de 1991, Bogotá 1994, S. 132; Chaín Lizcano (Fn. 8), S. 71 (103ff).

22 SCC Nr.C-074/93 vom 25.02.1993, in: RCC 1993 Nr.11, S. 27ff; i.d.S. auch SCC Nr.C-006/93 vom 18.01.1993, in: RCC 1993 Nr.1. S. 19ff; SCC Nr.C-216/93 vom 09.06.1993, in: RCC 1993 Nr.30, S. 25ff; jeweils unter besonderer Berücksichtigung der Eigentums- und Unternehmensfreiheit. 
sung $^{23}$. Die Verfassung soll ein entsprechendes Gesellschaftsmodell vorzeichnẹn, nämlich ein positives Programm, welches den Gesetzgeber auf die Verwirklichung einer gerechten politischen, wirtschaftlichen und sozialen Ordnung verpflichtet. ${ }^{24}$

Diese Konzeption spiegelt sich zunächst in einem Fächer staatlicher Lenkungsinstrumente wider, die ihrerseits nach Interventionsstufen differenziert werden ${ }^{25}$. Folgerichtig ist ferner eine den genannten Lebensbereichen entsprechende Gliederung der subjektiven Rechte. Diese sollen die Privaten "jenseits ihrer persönlichen Entwicklung und Menschenwürde in ihrer gesellschaftlichen Dimension, das heißt in der Spannung Individuum-Gesellschaft erfassen" ${ }^{26}$. Daher differenziert der Verfassungsgeber bereits formell die im zweiten Titel der Verfassung aufgeführten Rechte und Pflichten in "Grundrechte" (1. Kapitel) sowie in "Sozial-, Wirtschafts- und Kulturrechte" (2. Kapitel). Die kolumbianische Lehre überhöht diese Differenzierung - durchaus folgerichtig angesichts der drängenden sozialen Fragen und verleiht den Rechten einen unterschiedlichen Grad gesellschaftlicher Pflichtigkeit. Demnach dienen die überwiegend zum klassischen Kanon gehörenden Grundrechte als "privatnützige Rechte" (derechos particulares) quasi vorstaatlich dem Freiheitsgebrauch des Inhabers. Das betrifft die Bereiche Lebens- und Persönlichkeitsschutz, das Gleichheitsgebot, die Gewissens-, Glaubens-, Meinungs-, Presse- und Berufsfreiheit sowie die Justizgrundrechte. Dagegen stehen die Sozialrechte als "fremdnützige Rechte" (derechos altruistas) unter dem Primat ihrer gesellschaftlichen Bindung. Die Ausübung ist von vornherein auf dasjenige $\mathrm{Maß}$ begrenzt, in dem der individuelle Gebrauch zugleich den Interessen der Allgemeinheit nützt ${ }^{27}$. Das Verfassungsgericht übernimmt für diese Differenzierung das Begriff spaar "Rechte der ersten" und "Rechte der zweiten Generation" ${ }^{28}$. Im Kern handelt es sich um den gleichen Gedanken, nämlich die unterschiedliche Schutzwürdigkeit der Verfassungsrechte, je nach der gesellschaftlichen Relevanz der individuellen Befugnisse. Diese Trennung nach Lebensbereichen, die unterschiedlich stark entweder als Ausdrucksformen persönlicher Freiheit oder des solidarischen Handelns gegenüber der Gesellschaft aufgefaßt werden, folgt dem Vorbild der spanischen Verfassung von $1978^{29}$. Kennzeich-

SCC vom 17.6.1992, in: Jurisprudencia y Doctrina 1992, S. 727 (730f).

Näher für die Wirtschaftslenkung Chaín Lizcano (Fn. 8), S. 71 (122ff); für die Umwelt- und Entwicklungsplanung Timmermann (Fn. 6), S. 222ff.

SCC Nr.T-002/92 (Fn. 23), S. 183 (187).

Ernesto Peña Qiñones: La propiedad. Su evolución historíca en el régimen constitucional colombiano, in: Univérsitas Nr.83 (1992), S. 411 (462f).

SCC Nr.T-506/92 vom 21.8.1992, in: GCC 1992, S. 537 (541f).

Tulio Eli Chiuchilla Herrera: Qué son y Cuales son los Derechos Fundamentales?, in: Estudios de Derecho Nr.127 (1997), S. 37 (60f). Der erste spanische Verfassungstitel ("Von den Grundrechten und Grundpflichten") zerfällt im Unterschied etwa zum Bonner Grundgesetz in eine erste Sektion mit den grundrechtlich geschützten Freiheitsrechten (Art. 15-29 CE) und in eine zweite Sektion 
nend für das Bedürfnis nach einer erschöpfenden Intepretation im kolumbianischen Fall ist die Verbindung gesellschaftspolitischer Kriterien mit einem historischen Ansatz: Während die Abwehr- und Schutzrechte der ersten, liberalstaatlichen Verfassungsepoche noch eine möglichst unbeeinträchtigte Entfaltung der Persönlichkeit zugrundelegen konnten, stehen die Rechte der zweiten Generation in einem Zusammenhang mit dem jüngeren Leistungsund Versorgungsstaat. Diese zweite Kategorie müsse eine gleiche Teilhabe aller Bürger am gesellschaftlichen und wirtschaftlichen Fortschritt gewährleisten, das heißt auch die Grundbedürfnisse benachteiligter Gruppen befriedigen helfen ${ }^{30}$. Dazu gehören nicht nur die Tariffreiheit und das Streikrecht (Art. 55 bzw. 56 CP), die systematisch von der Versammlungs- (Art. $37 \mathrm{CP}$ ), Vereinigungs- und Koalitionsfreiheit (Art. 38, $39 \mathrm{CP}$ ) getrennt wurden $^{31}$. Entsprechend ihrer materiellen Bedeutung zählen hierunter auch die Wirtschaftsrechte Eigentum (Art. $58 \mathrm{CP}$ ) und Unternehmensfreiheit (Art. $333 \mathrm{CP}$ ), die aufgrund ihrer besonderen sozialen Pflichtigkeit nicht per se Bestandteil des Grundrechtekanons sind. ${ }^{32}$

\section{b. Die Abstufung}

In der Konsequenz einer förmlichen Differenzierung der Individualrechte liegt auch die Abstufung der subjektiven Gewährleistungen nach der unterschiedlichen Reichweite ihres Verfassungsschutzes. Gemäß der kolumbianischen Lehre sind Grundrechte im materiellen Sinne solche individuellen Befugnisse, die im Unterschied zu den Sozial- und Wirtschaftsrechten durch besondere Mechanismen (garantías reforzadas) einen verstärkten Rechtsschutz erfahren ${ }^{33}$. Zugleich kennzeichnen die Grundrechte in Anlehnung an die deutsche Lehre auch Elemente einer objektiven Wertordnung (un valor del ordenamiento), weshalb sie die Grundlage für staatliche Schutzpflichten bilden. ${ }^{34}$

aus minder geschützten Wirtschafts- und Sozialrechten (Art. 30-38 CE), zu denen auch das Eigentum und die Unternehmensfreiheit gehören.

Eduardo Cifuentes Muñoz: Derechos fundamentales e interpretación constitucional, in: Eduardo Cifuentes Muñoz/Libardo Rodríguez Rodríguez u.a. (Hg.): Nuevas corrientes del Derecho constitucional colombiano, Bogotá 1994, S. 5 (10f); Alfredo Manrique Reyes: La Constitución de la nueva Colombia, Bogotá 1991, S. 28f.

Zu den Schutzbereichen und Abgrenzungsfragen Camilo Velásquez. Turbay: Derechos y libertades fundamentales en la nueva Constitución, y otros aspectos de congreso y gobierno, in: Externado 1992 Nr.1, S. 37 (47ff).

Für das Eigentum SCC T-Nr.488/92 vom 11.8.1992, in: GCC 1992, S. 293 (294, 304); SCC Nr.C-374/97 vom 13.8.1997, in: Derecho Colombiano Nr.431 (1997), S. 403 (412); SCC Nr.C409/97 vom 28.8.1997, in: Foro Colombiano Nr.341 (1997), S. 466 (469).

34

Chiuchilla Herrera (Fn. 29), S. 37 (38f, 61).

Mit Bezug auf Leben und Gesundheit: SCC Nr.C-239/97 vom 20.5.1997, in: Derecho Colombiano Nr.427 (1997), S. 3 (17f), zur Verfassungsmäßigkeit des Art. 326 Strafgesetzbuch (Strafbarkeit der Sterbehilfe); und SCC Nr.C-309/97 vom 25.6.1997, in: Foro Colombiano Nr.341 (1997), S. 403 (420f), zur Pflicht der Fahrzeuginsassen, Sicherheitsgurte anzulegen. 
Zu den besonderen Schutzvorkehrungen der Verfassung gehört zunächst die unmittelbare Wirkung der Grundrechte (aplicación immediata). Demnach bedarf keines der in Art. 85 $\mathrm{CP}$ genannten Rechte eines vermittelnden Gesetzes, um gegenüber den öffentlichen Gewalten vor Eingriffen zu schützen. Aus Art. 85 ergibt sich aber zugleich auch eine Korrektur der förmlichen Einteilung, da zahlreiche Artikel des Grundrechtekapitels nicht in dieser abschließenden Aufzählung enthalten sind: So das Recht auf Frieden (Art. 22), das Recht auf Arbeit (Art. $25 \mathrm{CP}$ ), das Recht auf Asyl (Art. $36 \mathrm{CP}$ ) sowie die Vereinigungs(Art. $38 \mathrm{CP}$ ) und Koalitionsfreiheit (Art. $39 \mathrm{CP}$ ). Dagegen erzeugt die Versammlungsfreiheit (Art. $37 \mathrm{CP}$ ) eine unmittelbare Bindung der öffentlichen Gewalten und zählt damit auch im materiellen Sinne zu den Grundrechten.

Zugleich entfalten die Grundrechte nach kolumbianischer Lehre eine unmittelbare Drittwirkung. Sie garantieren nicht nur eine wirksame Abwehr gegenüber den öffentlichen Gewalten, sondern auch gegenüber anderen Privaten einzuhaltende Ordnungsgrundsätze für den sozialen Kontakt im entsprechenden Lebensbereich. So ist beispielsweise mit der Ächtung der Todesstrafe (Art. 11 S.2 CP) und anderer grausamer Sanktionen (Art. 12 CP) auch das unmittelbar bindende Verbot der Tötung, Entführung und Folterung der Mitmenschen ausgesprochen ${ }^{35}$. Das Verfassungsgericht begründet die Anerkennung einer unmittelbaren Drittwirkung der Grundrechte mit der Fortentwicklung des liberalen Staatsverständnisses zur Konzeption des modernen Sozialstaates. Der politische Wandel impliziere eine neue Auffassung von den bürgerlichen Pflichten, derzufolge das Gemeinwesen auf der gegenseitigen Solidarität beruhe ${ }^{36}$. Diese Besonderheit im Unterschied zu vielen anderen Verfassungsordnungen der westlichen Hemisphäre entspricht dem Bemühen der Konstituante, die neue Verfassung jedem kolumbianischen Bürger als Rechts- und Verhaltensgrundlage näher zu bringen. Für einzelne Bereiche, wie den Lebensschutz, lassen sich Verfassungsgeber und Verfassungslehre zudem von den inneren Bedrohungen des Gemeinwesens leiten. ${ }^{37}$

Ein weiterer privilegierter Schutz, den nur Grundrechte erfahren, gründet in der kolumbianischen Schrankenlehre. Im Anschluß an die deutsche Lehre unterliegt die Ausübung vorbehaltloser Grundrechte allein verfassungsimmanenten Schranken. Eine Beschränkung des Grundrechtsgebrauchs ist demnach nur dann zulässig, wenn nach sorgfältiger Abwägung

35

36

37

Velásquez. Turbay (Fn. 31), S. 37 (37, 39); ferner Ley 282/1996 vom 6.6.1996 ("por la cual se dictan medidos tendientes a erradicar algunos delitos contra la libertad personal"), in: Derecho Colombiano Nr.415 (1996), S. $7 \mathrm{ff}$.

SCC Nr.T-477/95 vom 23.10.1995, in: Foro Colombiano Nr.318 (1995), S. 520 (564), zur Pflicht der Eltern, Menschenwürde, Persönlichkeitsrecht und Entfaltungsfreiheit ihres Kindes zu wahren, das einer Geschlechtsumwandlung unterzogen worden war.

Aufschlußreich Velásquez Turbay' (Fn. 31), S. 37 (41), der die seit 1852 endgültig verbotene Sklaverei in einen Zusammenhang mit den aktuellen Fragen Menschenhandel und Bürgerkrieg stellt. 
der Ausgleich mit kollidierenden Grundrechten oder mit Grundwerten von Verfassungsrang dies erfordert ${ }^{38}$. So ist als ungeschriebene Begrenzung der Meinungs- und Pressefreiheit (Art. 20 Abs. 1 Alt. 1 bzw. Alt. 2 CP) in subjektiver Hinsicht der Ehrschutz (Art. 21 CP) und in objektiver Hinsicht der öffentliche Friede (Art. 22 CP) anerkannt ${ }^{39}$. Der Ehrschutz bildet mit den übrigen Ausprägungen des Persönlichkeitsschutzes (Art. $15 \mathrm{CP}$ ) auch eine wichtige verfassungsimmanente Schranke der Informationsfreiheit (Art. 20 Abs. 1 Alt. 3 $\mathrm{CP})^{40}$. Wie gerade in der ausführlichen Behandlung der informationellen Selbstbestimmung (Art. $15 \mathrm{CP}$ ) deutlich wird, suchte der Verfassungsgeber nach einem angemessenen Ausgleich der verschiedenen kollidierenden Interessen im Bereich der Informationsbeschaffung und -verwertung, nicht ohne auch die zunehmend gefährdete Position des Einzelnen angesichts verbesserter technischer Möglichkeiten zur Datensammlung und -speicherung schützen zu wollen. ${ }^{41}$

Für die verbleibenden, mit einem Gesetzesvorbehalt (reserva de ley) versehenen Grundrechte konstituieren Art. 152a und Art. 153 CP höhere Anforderungen an Eingriffsregelungen als für Beschränkungen der übrigen Verfassungsrechte. Soweit durch ein Gesetz Grundrechtspositionen berührt werden, erfordert dessen Verabschiedung die absolute Mehrheit der (anwesenden) Kongreßmitglieder, wobei das gesamte Verfahren innerhalb einer Legislaturperiode abgeschlossen sein muß (Art. 153 Abs. $1 \mathrm{CP}$, sog. ley estatuaria) ${ }^{42}$. Schließlich gilt für alle Grundrechte, daß sie im Ausnahmezustand nicht durch Notstandsgesetze suspendiert werden können (Art. $214 \mathrm{Nr} .2 \mathrm{CP}$ ), was dagegen für die minder geschützten Wirtschafts- und Sozialrechte zulässig ist. ${ }^{43}$

SCC Nr.T-3/95 vom 16.1.1995, in: Foro Colombiano Nr.308 (1995), S. 150 (154f), hinsichtlich der Gewissens- und Glaubensfreiheit (Art. 18f CP); ferner SCC Nr.C-239/97 (Fn. 34), S. 3 (18), mit Bezug auf den Lebensschutz (Art. 11 CP); für die Literatur Cifuentes Muñoz. (Fn. 30), S. 5 (15f); weitere vorbehaltlos gewährleistete Grundrechte sind u.a. die körperliche Unversehrtheit (Art. 12), die Wahrung der Rechtspersönlichkeit (Art. 14), das Verbot der Sklaverei und Knechtschaft (Art. 17).

Näher Carlos Villalba Bustillo: El soporte jurídico de la libertad de prensa en Colombia, in: Externado 1995 Nr.1, S. 23 (25f), ders. auch zu den Ansprüchen auf Unterlassung, Widerruf und Schadensersatz bei Ehrverletzungen (S. 26f).

SCC Nr.T-551/94 vom 2.12.1994, in: Foro Colombiano Nr.307 (1995),S. 92 (96); Nelson Remolina Angarita: El Habeas Data en Colombia, in: Revista de Derecho Privado Nr.15 (1994), S. 185 (210f), m.w.N. zur Rechtsprechung.

41 Remolina Angarita (Fn. 40), S. 185 (191); María Fernanda Guerrero M.: Una aproximacíon al análisis del derecho a la intimidad, in: Univérsitas Nr.84 (1993), S. 369 (37lff).

Zur Reichweite der leyes estatuarias SCC Nr.C-350/97 vom 29.7.1997, in: Foro Colombiano Nr.339 (1997), S. 203 (210); SCC Nr.C-374/97 (Fn. 32), S. 403 (409).

Die Voraussetzungen sind ausführlich in Art. 212-215 der Verfassung geregelt. 
Die wohl wichtigste Differenzierung des Rechtsschutzes ist mit der Anwendung des neuen Rechtsbehelfs acción de tutela verbunden. Die tutela besteht in einem bevorzugten, summarischen Verfahren vor den Verwaltungsgerichten, deren zweite Instanz den Antrag an das Verfassungsgericht weiterleitet (Art. 86 Abs. 2 CP). Gegenstand der Überprüfung sind bevorstehende oder andauernde Beeinträchtigungen durch die öffentlichen Gewalten, sofern sie konkret-individueller Natur sind ${ }^{44}$. Anders als nach Art. 53 Abs. 2 der spanischen Verfassung richtet sich der privilegierte Gerichtsschutz gemäß der kolumbianischen Lehre nicht von vornherein nach der systematischen Einordnung der Verfassungsrechte, sondern nach ihrer Qualifizierung als Grundrechte im übrigen (Art. 86 Abs. $1 \mathrm{CP}$ ). Daher erfahren diejenigen Individualrechte des Grundrechtekapitels den Schutz der tutela nicht, die auch nicht unter den in Art. 85 CP unmittelbar bindenden Garantien aufgeführt werden, so das Recht auf Arbeit $(25 \mathrm{CP})^{45}$. Andererseits ist bei Verletzung weiterer, auch außerhalb des Grundrechtekapitels genannter Verfassungsrechte die tutela statthaft. Dies betrifft das Recht der Eltern, über die Zahl ihrer Nachkommen zu entscheiden (Art. 42 Abs. 7 CP), ferner die Gleichbehandlung der Geschlechter (Art. 43 Abs. 1 CP) und die in Art. 44 Abs. 1 CP genannten Grundrechte der Kinder, unter anderen auf Leben, körperliche Unversehrtheit und freie Meinungsäußerung ${ }^{46}$. Allerdings wird hierdurch im Ergebnis keine tatsächliche Ausweitung des Gerichtsschutzes bewirkt. Denn der Verfassungsgeber hat im Grunde lediglich die allgemeinen grundrechtlichen Garantien für den speziellen Regelungsbereich der Familie unterstrichen ${ }^{47}$. Hierin zeigt sich die insgesamt charakteristische Neigung der Konstituante, durch detaillierte und oftmals wiederholende Formulierungen im Verfassungstext eine umfassende, möglichst vollständige thematische Behandlung zu erreichen. Diese Tendenz ist weniger den klassischen Funktionen des Grundrechtsschutzes - Abwehr, Teilhabe und Leistung - geschuldet. Sie ist vielmehr als ein Appell an die Gesellschaft zu verstehen, verbunden mit dem pädagogischen Bemühen, die Verfassung als eine anschau-

Zur Reichweite des Schutzes SCC Nr.T-004/95 vom 16.1.1995, in: Foro Colombiano Nr.308 (1995), S. 157 (163f); SCC Nr.C-243/96 vom 30.5.1996, in: Foro Colombiano Nr.325 (1996), S. 32 (35); José María Forero B.: Los derechos fundamentales y su desarrollo jurisprudencial, Bogotá 1994, S. 86ff; für die Anfechtung eines Parlamentsgesetzes oder eines (generell-abstrakten) Regierungsaktes im Gesetzesrang ist die Normenkontrollklage der Bürger gem. Art. $241 \mathrm{Nr} .4$ und $5 \mathrm{CP}$ statthaft; näher Jorge Leyva Valenzuela: La acción pública de inconstitucionalidad y la inconstitucionalidad por omisión legislativa, in: Univérsitas Nr.90 (1996), S. 169 (171ff). Allerdings besteht bzgl. Art. 25 CP Uneinigkeit, m.w.N Chiuchilla Herrera (Fn. 29), S. 37 (63ff). Chiuchilla Herrera (Fn. 29), S. 37 (64f); SCC Nr.T-477/95 (Fn. 36), S. 520 (561 f), bzgl. Art. 44 $\mathrm{CP}$.

Dies belegt SCC Nr.C-625/96 vom 21.11.1996: Aus dem Zusammenhang (sog. Konnexität) mit der Handlungs- und Entfaltungsfreiheit (Art. $16 \mathrm{CP}$ ) ergibt sich ein "Grundrecht" der Kinder auf Freizeit (Art. 44 CP), in: Foro Colombiano Nr.326 (1996), S. 103 (110). 
liche Darstellung und erzieherische Grundlage der staatsbürgerlichen Rechte und Pflichten aller Kolumbianer zu gestalten. ${ }^{48}$

\section{c. $\quad$ Korrekturen der Schutzsystematik}

Fast zwangsläufig ergeben sich mit der Tendenz zur Systematisierung und Abstufung des Rechtsschutzes Abgrenzungsschwierigkeiten, die Rechtsprechung und Literatur zu Korrekturen der Verfassungssystematik veranlaßt haben. In der Literatur wird darauf hingewiesen, daß die generelle Ausnahme der Wirtschafts- und Sozialrechte vom Grundrechtsschutz mit Blick auf das Rechtsstaatsprinzip problematisch sei. Denn Art. 2 Abs. 2 CP verpflichte die öffentlichen Gewalten, alle Verfassungsrechte zu schützen und hebe dabei die Gewährleistung des Vermögens hervor ${ }^{49}$. Damit ist ein minderer Schutz insbesondere des Privateigentums und der Unternehmensfreiheit kaum zu vereinbaren; zumal sowohl die Formulierung des Art. 58 Abs. $1 \mathrm{CP}$ als auch jene des Art. 333 Abs. $1 \mathrm{CP}$ die liberale Grundrechtskonzeption einer Abwehr gegen staatliche Eingriffe in Eigentum und Freiheit aufgreif en ${ }^{50}$. Hinzu kommt der enge Bezug der Wirtschaftsrechte zu jener für die kolumbianische Gesellschaft so wichtigen Frage der materiellen Existenzsicherung. Diese Motive haben das kolumbianische Verfassungsgericht zu einer weiteren Differenzierung veranlaßt. Es erkennt eine zusätzliche Kategorie der Grundrechte an, nämlich solche "mittelbarer Anwendung" (Derechos fundamentales de aplicación indirecta). Hierzu zählen diejenigen Wirtschaftsund Sozialrechte, die in einer besonders nahen Verbindung mit anderen, stets unmittelbar anwendbaren Grundrechten stehen, etwa weil sie dem Lebens- und Gesundheitsschutz oder der Verwirklichung materieller Gleichheit dienen ${ }^{51}$. So genießt das Privateigentum immer dann den vollen grundrechtlichen Schutz, insbesondere den der acción de tutela, wenn es dem Erhalt der materiellen Existenzgrundlage dient, ohne deren Bewahrung die Gleichbehandlung und eine würdevolle Lebensweise der Betroffenen berührt wären. Diese Einschätzung soll das Gericht nur im Einzelfall und unter Berücksichtigung der konkreten Bedürfnisse des Rechtsinhabers treffen, wobei nicht nur die Mindestexistenz einer Familie,

Vgl. auch die letzte Norm des Grundrechtekapitels: Art. 41 CP verpflichtet den kolumbianischen Staat, die Verfassung und die demokratischen Regeln zum Gegenstand der Unterweisung in allen Bildungseinrichtungen zu machen, worin eine Grundvoraussetzung politischer Teilhabe und Willensbildung erkannt wird, und zwar in unmittelbarer Nähe zum aktiven und passiven Wahlrecht (Art. 40 CP); dazu Velásquez. Turba)' (Fn. 31), S. 37 (51); ferner Humberto de la Calle Lombano: Derechos en la reforma constitucional, in: Externado 1991 Nr.1, S. 3 (7f), mit dem Hinweis auf

49

50

5

Eine Verbindung des Vermögensschutzes zum Gleichheitssatz konstruiert etwa SCC Nr.T-506/92 (Fn. 28), S. 537 (542). 
sondern auch die Gefährdung eines Betriebes zu einer besonderen Schutzbedürftigkeit führen können.

Im Ergebnis hat die flexible Handhabung des Grundrechtsschutzes vor allem zwei Folgen. Die Richter müssen einerseits die privaten und gesellschaftlichen Belange, welche mit der Existenzsicherung verbunden sind, schon für die Anwendbarkeit des privilegierten Grundrechtsschutzes berücksichtigen, nicht erst in der materiellen Prüfung. Damit wird die Reichweite des Rechtschutzes zum Gegenstand schwer überprüfbarer Einzelfallentscheidungen und ist abhängig von den sich fortwährend ändernden gesellschaftlichen Bedingungen. Andererseits ist der Rechtsschutz nunmehr nicht allein durch das Gewicht der Allgemeinwohlbelange begrenzt; er relativiert sich auch je nach der individuellen Nutzung der Güter. Demnach genießen die Nutzung und Verfügung über private Güter einen minderen Schutz, sobald sie über eine existenzsichernde Bedarfsdeckung hinausgehen. Die Unterscheidung zwischen Luxusgebrauch und Mindestversorgung wird zu einer unmittelbar den subjektiven Rechtsschutz beeinflussenden Kategorie erhoben.

\section{d. Die Übernahme einzelner Rechtsfiguren}

Auf einer Linie mit der Korrektur der eigenen Schutzsystematik liegt auch die besondere Anwendung und Interpretation solcher Institute, die in anderen Rechtsordnungen ausgeprägt wurden. So hat das kolumbianische Verfassungsgericht zwei Elemente der deutschen Lehre von den sogenannten Schranken-Schranken aufgenommen, und zwar über den dogmatischen Umweg der spanischen Lehre: In subjektiv-rechtlicher Hinsicht anerkennt das Gericht einen grundsätzlich unantastbaren Kern als Merkmal der Grundrechte (contenido o núcleo esencial) ${ }^{53}$. Diese Wesensgehaltsgarantie gründet in Anlehnung an die deutsche Verfassungslehre $^{54}$ in der unverletzlichen Menschenwürde (Art. $1 \mathrm{CP}$ ), wie sie nach kolumbianischer Lehre beispielsweise der Handlungsfreiheit (Art. $16 \mathrm{CP}$ ) ${ }^{55}$, dem Ehrschutz (Art. $21 \mathrm{CP}$ ) oder der Meinungsfreiheit (Art. 20 Abs. $1 \mathrm{CP}$ ) zugrundeliegt ${ }^{56}$. Objektivrechtlich stehen bestimmte Verfassungsinstitute unter dem besonderen Schutz bestimmter

Näher SCC Nr.T-506/92 (Fn. 28), S. 537 (542f); SCC Nr.T-381/93 vom 14.9.1993, in: Jurisprudencia y Doctrina 1993, S. 1189 (1190): Das Verfassungsgericht bejahte den Grundrechtsschutz und die Anwendung der tutela gegen eine Inspektion der Steuerbehörde, welche die Brauerei "Bavaria" zur Aushändigung von Braurezepten veranlaßt hatte. 
Einrichtungsgarantien, wie Ehe und Familie (Art. 42 Abs. 2 CP), Erbfolge (Art. 62 Abs. 1 $\mathrm{CP}$ ), Privateigentum (Art. 58 Abs. 1 CP) und Unternehmensfreiheit (Art. 333 Abs. 1 CP). Hiermit ist - ebenfalls nach dem Vorbild der deutschen Lehre - die Wahrung eines Mindestbestandes individueller Befugnisse verbunden, der nicht zur Disposition des Gesetzgebers steht ${ }^{57}$. Ergänzend soll auch die Wesensgehaltsgarantie mindestens diejenigen Befugnisse und Handlungsmöglichkeiten des Rechtsinhabers wahren, die das betreffende Recht typenhaft kennzeichnen und erst eine konkrete, wirksame Rechtsausübung erlauben. ${ }^{58}$

Diese Kriterien legt das Verfassungsgericht allerdings recht weit und je nach den Anforderungen des Allgemeinwohls an die Rechtsinstitute sehr flexibel aus. Dies wird am Beipiel der Wirtschaftsrechte Eigentum und Unternehmensfreiheit deutlich. Das Verfassungsgericht unterstreicht, daß der Bereich der individuellen Befugnisse in erster Linie durch die gesellschaftlichen Anforderungen definiert ist. So soll die Einrichtungsgarantie des Eigentums nicht notwendig auch dessen Bewahrung in allen Fällen gewährleisten. Zu erhalten sei stets nur jenes kleinste Maß wirtschaftlicher Nutzung und Verfügung, welches das "soziale Gewissen" als ausreichend empfinde, um ein privates Interesse anzuerkennen ${ }^{59}$. Auf dieser Linie bewegt sich auch die ständige Rechtsprechung zur entschädigungslosen Auslöschung des Grundeigentums (extinción del dominio) ${ }^{60}$. Die Anwendung dieses Instituts wurde bisher weder nach Maßgabe der Wesensgehaltsgarantie noch des gleichfalls anerkannten Übermaßverbotes als ein Verstoß gegen Art. 58 Abs. 1 oder Art. 333 Abs. 1 CP gewertet. ${ }^{61}$ Im Ergebnis soll der Gesetzgeber einen weiten Spielraum haben, damit er die wirtschaftliche und gesellschaftliche Bedeutung der Rechtsinstitute an die jeweiligen äußeren Bedingungen anpassen und immer neu interpretieren kann. Dieser Gestaltungsraum ist eine Grundbedingung für ein Land auf dem Wege der wirtschaftlichen Entwicklung. So ist auch das immer wieder erneuerte Bekenntnis zu verstehen: "Sobald das Privateigentum nicht mehr den gesellschaftlichen Anforderungen entspricht, ist der Gesetzgeber gehalten, eine andere Form des materiellen Erwerbes zu bestimmen" ${ }^{162}$. Dabei zielte die Rechtsprechung

Näher SCC Nr.C-006/93 (Fn. 22), S. 36f, zum Mindestbestand des Privateigentums: individuelle Nutzung (facultad de goce) und Verfügung (facultad de disposición) über das betreffende Gut. SCC Nr.T-426/92 vom 24.6.1992, in: RCC 1992, S. 16f; im Anschluß an das spanische Verfassungsgericht: STC Nr.37/87 vom 26.3.1987, in: Boletín Oficial del Estado (B.O.E.) Nr.89 vom 14.4.1987, Rdnr.2; STC Nr.170/89 vom 19.10.1989, in: B.O.E. Nr.267 vom 7.11.1989, Rdnr.8b). SCC Nr.C-006/93 (Fn. 22), S. 37. Vgl. SCC Nr.C-374/97 (Fn. 32), S. 403 (423), und SCC Nr.C-409/97 (Fn. 32), S. 466 (470), mit dem Hinweis auf die "gesellschaftliche" bzw. "kollektive Moral" als Grenze des Eigentumsrechts.

Zur Minengesetzgebung SCC Nr.C-006/93 (Fn. 22), S. 41 ff; und SCC Nr.C-216/93 (Fn. 22), S. $22 \mathrm{ff}$.

62 So die Rechtsprechung seit dem Urteil des Staatsrates, SCSJ vom 10.3.1938, in: Jurisprudencia y Doctrina 1982, S. 448 (449); ferner SCSJ vom 11.8.1988, in: JD 1988, S. 907 (914); und SCC Nr.C-006/93 (Fn. 22), S. 19. Die Aussage geht zurück auf Léon Duguit: Traité de droit constitutionnel, Bd.3, Paris 1923, S. 618. 
ebensowenig wie die Konstituante auf eine grundlegende Änderung der Vermögensverhältnisse oder auf eine weitreichende Vergesellschaftung ab. Sie will aber vermeiden, daß durch eine doppelte, nämlich institutionelle wie individuelle Garantie, der Gestaltungsspielraum des Gesetzgebers von vornherein beschränkt und die Vermögensverteilung auch verfassungsrechtlich unverrückbar wird. Dies muß um so mehr gelten, je deutlicher die faktische Eigentumsordnung durch große Ungleichheit gekennzeichnet ist und je stärker der Staat selbst an der Sicherung des Existenzminimums seiner Bürger interessiert ist ${ }^{63}$. Vor diesem Hintergrund wird auch die Begründung des kolumbianischen Verfassungsgerichts für die zahlreichen Eingriffsmöglichkeiten in die wirtschaftlichen Befugnisse der Privaten verständlich: Die Rechtsausübung soll der ganzen Gesellschaft dienen, und zwar

in einem um so stärkeren Maße, wie es um die Nutzung wirtschaftlicher Güter geht. ${ }^{64}$

\section{Die Abgrenzung der subjektiven Rechte}

Die besonderen Charakteristika des Verfassungschutzes subjektiver Rechte finden ihre Entsprechung in der Abgrenzung zu zwei anderen Kategorien der kolumbianischen Verfassung. In ihnen spiegelt sich nicht nur die Neigung zur Systematisierung und Überhöhung, verbunden mit der Gefahr zur Auflösung eben dieser subtilen Klassifizierung. Auch die Verankerung neuer Rechtsfiguren in der Verfassung von 1991 steht unter dem Vorzeichen wirtschaftlicher Entwicklung und drängender sozialer Anliegen.

Von den Individualrechten im herkömmlichen Sinne, die eine einheitliche und ausschließliche Befugnis des Rechtsinhabers kennzeichnet, unterscheiden sich zunächst die gemeinschaftlichen Rechte (derechos comunitarios). Das sind nicht-ausschließliche Rechte, die in gemeinsamer Rechtsträgerschaft mit anderen, in natürlicher oder rechtlicher Gemeinschaft verbundenen Personen ausgeübt werden ${ }^{65}$. Beispiele sind das gemeinschaftliche Grundeigentum der Eingeborenen eines Reservates (Art. 63, 329 Abs. 2 CP) und die genossenschaftlichen Landwirtschaftsbetriebe (Art. $64 \mathrm{CP}$ ). Träger des Rechts ist die betreffende Gemeinschaft, nicht das einzelne Rechtssubjekt. Das Ausscheiden aus der Gemeinschaft beläßt das Recht im übrigen unberührt, eine individuelle Einwirkung auf den Bestand des

Hierzu Adalbert Podlech: Eigentum - Entscheidungsstruktur der Gesellschaft, in: Der Staat Nr.15 (1976), S. 31 (32f).

64 SCC Nr.C-006/93 (Fn. 22), S. 37; SCC Nr.C-216/93 (Fn. 22), S. 26f.

65

Hernán Valencia Restrepo: La nueva Constitución colombiana y la teoría general de derecho civil, in: Estudios de Derecho Nr.117f (1991), S. 75 (80), der etwas mißverständlich dennoch von subjektiven Rechten spricht. 
Rechtes im Sinne einer Übertragung, Aufhebung oder Belastung ist ausgeschlossen ${ }^{66}$. Nur in Ausnahme- und Härtefällen kann dem einzelnen ein Abwehranspruch erwachsen ${ }^{67}$. Auch in gesellschaftspolitischer Hinsicht unterscheidet sich dieser Ansatz von der Konzeption der sozialstaatlich inspirierten Zugangs- und Teilhaberechte. Maßgeblich sind die viel weiter reichenden Anliegen einer umfassenden Boden- und Gesellschaftsreform einerseits, bei Sicherung der bedrohten Existenzgrundlage andererseits. So steht Art. $64 \mathrm{CP}$ nicht in erster Linie mit der Sozialbindung des Eigentums (Art. 58 Abs. 2 CP) in einem Zusammenhang, sondern mit der staatlichen Förderung kollektiver Eigentumsformen (Art. 58 Abs. 3 CP). Ganz in diesem Sinne hält es das Verfassungsgericht auch für vereinbar mit den Zielen der Konstituante, dảß eine "Demokratisierung" des Eigentums und eine fortschreitende Teilhabe der Bürger an den Produktionsmitteln durch gezielte Enteignungen realisiert werden, und zwar auch ohne eine Entschädigung des Rechtsinhabers (Art. 58 Abs. $5 \mathrm{CP}){ }^{68}$

Die zweite der von den subjektiven Rechten abzugrenzenden Kategorien nimmt weitere sozialpolitische Anliegen auf und verbindet sie mit globalen, überwiegend ökologischen Zukunftsfragen einer "Dritten Generation", worin thematisch die Abgrenzung zu den vorrangigen Aspekten Abwehr und Teilhabe der subjekten Rechte zu sehen ist. Das am stärksten beachtete unter den auch Kollektiv- und Umweltrechten genannten Normen im gleichnamigen dritten Kapitel ist das Recht aller Menschen auf den Genuß einer heilen Umwelt (Art. 79 Abs. 1 CP). Das Hauptanliegen dieses wie auch der übrigen Kollektivrechte (oder auch Drittgenerations- bzw. Solidaritätsrechte) wird darin erblickt, bestimmte Werte, wie Solidarität, Entwicklung und Umweltschutz, aufgrund ihrer besonderen Gefährdung grundrechtlich zu verstärken. ${ }^{69}$

Die mangelnde systematische wie inhaltliche Konsequenz zeigt sich jedoch mit einem Blick auf die weiteren Kollektivrechte. Auf der einen Seite zählen zu dieser Kategorie auch subjektiv-rechtlich formulierte Normen der beiden ersten Kapitel. Das betrifft das Recht auf

66

Dazu Jesús Vallejo Mejía: Apuntes sobre el régimen de la propiedad privada y la iniciativa privada en la Constitución de 1991, in: Revista de la Facultad de Derecho y Ciencias Políticas de la Universidad Pontificia Bolivariana Nr.91 (1990), S. 47 (55ff).

67 SCC Nr.T-254/94 vom 30.5.1994, in: Foro Colombiano Nr.302 (1994), S. 176 (185f), zum eigentumsähnlichen Schutz eines Eingeborenen aus Art. 34 CP (Konfiskationsverbot), der mit dem Stammesausschluß seine landwirtschaftliche Existenzgrundlage verloren hätte.

68

SCC Nr.C-006/93 (Fn. 22), S. 29f; dies entspricht den Positionen der Unterkommission Eigentum im Rahmen der fünften Verfassungskommission, Informe de Ponencia, in: Gaceta Constitucional Nr.46 vom 15.4.1991, S. 25f. Problematisch ist die entschädigungslose Enteignung nicht zuletzt mit Blick auf das internationale Recht; rechtfertigend Amparo Alicia Oviedo Arbeláez: Propiedad 69 privada, sus garantías y limitaciones en Colombia, in: Univérsitas Nr.90 (1996), S. 203 (212ff).

I.d.S. SCC Nr.T-451/92 vom 10.7.1992, in: GCC 1992, S. 374 (379); Manrique Reyes: (Fn. 30), S. $28 \mathrm{f}$. 
Frieden (Art. $22 \mathrm{CP})^{70}$, das Recht aller Bürger auf soziale Sicherheit (Art. 48 Abs. $2 \mathrm{CP}$ ) und auf Zugang zur Gesundheitsfürsorge (Art. 49 Abs. 1 S.2 CP) ${ }^{71}$. Andererseits faßt das dritte Kapitel typische Staatsaufgaben unter die Kategorie der Kollektivrechte. Das gilt für die Umweltplanung im Sinne einer nachhaltigen Entwicklung (Art. 80 Abs. 1 CP), worunter ein bindender Verfassungsauftrag zur Lenkung aller privaten und öffentlichen Aktivitäten mit Auswirkungen auf die Nutzung und den Zustand natürlicher Ressourcen zu verstehen ist ${ }^{72}$. Ferner gehören hierzu die Pflicht des Staates zur Wahrung des öffentlichen Raumes (Art. 82 Abs. $1 \mathrm{CP}$ ) und die Beteiligung der öffentlichen Hand am Mehrwert (Art. 82 Abs. $2 \mathrm{CP}$ ), der für private Grundstücke aus der Bebauungsplanung erwächst. Tatsächlich vermitteln die Kollektivrechte selbst keine subjektiv-rechtlichen Befugnisse. Vielmehr handle es sich um "überindividuelle Rechte" (derechos supra-individuales), deren Träger die Gemeinschaft sei und deren Ausübung den öffentlichen Gewalten überlassen werde ${ }^{73}$. Denn nur sie können jene Anliegen der Dritten Generation als Handlungsauftrag oder als Maßstab für Abwägung und Auslegung wahrnehmen. Im Ergebnis handelt es sich um Staatszielbestimmungen oder bloße Programmsätze ${ }^{74}$. Die Formulierung eines Teils dieser Rechte als individuelle Gewährleistungen soll zu einer Öffnung der Grundordnung beitragen. Am Ende dieser normativen Entwicklung könnten die Kollektivrechte den Rang subjektiver Rechte erhalten ${ }^{75}$. Um diese Perspektive zu unterstreichen hat das kolumbianische Verfassungsgericht einen spezifischen Gefährdungszusammenhang (Konnexität) anerkannt, über den jedenfalls mittelbar ein Anliegen der Dritten Generation den Grundrechtsschutz der tutela eröffnet: Dies gilt immer dann, wenn mit einer Beeinträchtigung eines solchen Belangs zugleich die Verletzung eines Grundrechtes verbunden ist; insbesondere des Rechts auf Leben (Art. $11 \mathrm{CP}$ ) oder auf körperliche Integrität (Art. $12 \mathrm{CP})^{76}$. Mit dieser Hilfskonstruktion ändert sich im Ergebnis nichts an der Einordnung der Kollektivrechte als Staatszielbestimmungen beziehungsweise als Programmsätze. Denn ein möglicher höherer Rechtsschutz beruht allein auf einer Verletzung des Klägers in einer anderen Gewährlei-

70

71

72

73

74

75

76

Std. Rspr., so bzgl. Art. 79 Abs. 1 CP SCC Nr.T-415/92 (Fn. 74), S. 326 (334ff); SCC Nr.T004/95 (Fn. 44), S. 157 (160f); ferner SCC Nr.SU-111/97 vom 16.3.1997, in: Derecho Colombiano Nr.426 (1997), S. 503 (510f), mit Bezug auf Art. 48 Abs. 2 und Art. 49 Abs. 1 S. 2 CP; ebenso für die Literatur Chiuchilla Herrera (Fn. 29), S. 37 (68). 
stung, nämlich in einem Grundrecht ${ }^{77}$. Dennoch ist die Figur der Drittgenerations- oder Kollektivrechte aufschlußreich als ein charakteristisches Beispiel für das Bestreben, Themen mit hohem politischen Stellenwert auf eine individualrechtliche Relevanz zuzuspitzen, hinter der aber die juristische Reichweite naturgemäß zurückbleibt.

\section{Ergebnis}

Die neuere kolumbianische Lehre vom Schutz der subjektiven Verfassungsrechte ist ein Spiegel der vielschichtigen sozialpolitischen Anliegen. So ist etwa die Zusammenstellung der zahlreichen unterschiedlichen, scheinbar gegensätzlichen Elemente zu erklären. Eine Konstante besteht jedoch darin, daß sich der Schutz der Individualrechte stärker als in den modernen Sozialstaaten nicht nur nach dem gesellschaftlichen Ausgleich bemißt, sondern auch nach der Notwendigkeit wirtschaftlicher Entwicklung. Die Allgemeinwohlbelange erhalten gegenüber den individuellen Interessen immer dann ein besonders hohes Gewicht, wenn wirtschaftliche Güter in Rede stehen. Aber auch die individuellen Befugnisse selbst werden danach unterschieden, in welchem Maß sie den Grundbedürfnissen dienen. Hinzu kommt, daß die Wirtschafts- und Sozialverfassung weitreichende Eingriffsziele und Instrumente formuliert. Sozialisierung und staatliche Förderung kollektiver Eigentumsformen sind Verfassungsaufträge, ebenso wie Wirtschaftslenkung und Entwicklungsplanung. Allerdings finden die visionären, jedoch häufig unerfüllbaren Maßgaben ihre Grenze in der Rechtswirklichkeit und in den nächstliegenden Bedürfnissen: Gerade die Existenzsicherung weiter Bevölkerungskreise, der Minderheitenschutz und der Nachholbedarf an wirtschaftlicher Entwicklung stärken eher die individuellen Befugnisse der Rechtsinhaber. Zugleich befördern sie pragmatische Lösungen in der Rechtsprechung sowie eine Tendenz zu Abstufung und Korrektur der Schutzsystematik. Die geringere dogmatische Konsequenz ist ein wichtiger Unterschied des kolumbianischen Verfassungsschutzes zur spanischen und deutschen Konzeption. Denn die Rechtswirklichkeit in Kolumbien prägt die Norminterpretation viel stärker als in den europäischen Vorbildern. Auch werden pragmatisçhe Lösungen und Einzelfallentscheidungen eher akzeptiert als in den zumeist prinzipientreueren Grundrechtslehren des alten Kontinents.

Festzuhalten ist ferner der Hang des kolumbianischen Verfassungsgebers zur vermeintlichen Vollständigkeit, der sich nicht nur im Umfang der einzelnen Verbürgungen zeigt. Eine Besonderheit ist auch die Formenvielfalt individueller und kollektiver Gewährleistungen. Die umfassende Behandlung der subjektiv-rechtlich erfaßten Lebensbereiche wird zusätzlich angereichert durch symbolische und appellative Elemente, die einer erzieherischen

77

Allerdings ergeben sich Weiterungen für den Rechtsschutz aus der Statthaftigkeit der tutela gegenüber Privaten und aus der extensiven Handhabung der Klagebefugnis, dazu Timmermann (Fn. 6), S. $218 \mathrm{ff}$. 
Motivation folgen. Die Verfassungsrechte sollen nicht nur Abwehr, Teilhabe und Zugang garantieren. Sie zielen darüber hinaus auf eine allgemeine Verhaltenssteuerung ab und sind zugleich Ausdruck einer hohen gesellschaftlichen Erwartungshaltung. Dies kommt in der unmittelbaren Drittwirkung der Grundrechte zum Ausdruck, aber auch in der Behandlung der globalen Anliegen, wie Frieden, Gesundheit und Umweltschutz, ferner in den Bestrebungen zu Gesellschaftsreform und materieller Umverteilung. Fast zwangsläufig hält aber die rechtlich-materielle Reichweite dem Symbolgehalt vieler Normen nicht stand. Auffallend ist nicht allein das Übergewicht der Wirtschafts-, Sozial- und Kollektivrechte gegenüber den klassischen Grundrechten. Auch scheint die politisch-soziale Rhetorik um so intensiver, je geringer die rechtliche Wirkung tatsächlich ist. Diese Tendenzen sind nicht ganz unproblematisch. Denn im Falle einer unzulänglichen Realisierung der Verfassungsziele wären eine weitere Schwächung der Identifikation mit dem Staat und eine noch geringere Beteiligung an den politischen Entscheidungsprozessen zu befürchten. Diese Umstände sollen aber die Bestrebungen des kolumbianischen Verfassungsgebers keinesfalls herabsetzen. Gerade die Verfassung von 1991 hat die öffentliche Diskussion um neue Themen bereichert und dem Bürger die Verfassung als justitiablen Kodex zweifellos nähergebracht. Dies belegt nicht zuletzt der häufige Gebrauch der acción de tutela. 


\title{
Neue Verfassungsstrukturen in Georgien
}

\author{
Von Wolfgang Gaul
}

"Die Unterzeichnung der Verfassung wird man nicht verhindern können", sagte Eduard Schewardnadse am 29. August 1995 nach einem Attentatsversuch auf ihn, das die für diesen Tag vorgesehene Unterzeichnung der neuen georgischen Verfassung nur um wenige Wochen verzögern konnte. Das mag in Erinnerung rufen, unter welchen Umständen und Vorzeichen die Verfassung in der Kaukasusrepublik entstand.

\section{A. Die Verfassungsgebung \\ I. Die Verfassung von 1921}

In Georgien wurde schon einmal, im Februar 1921, eine eigenständige Verfassung verabschiedet. An dieses Dokument konnte man anknüpfen, selbst wenn 1921, bereits vier Tage später, die GSSR, die "Georgische Sowjetische Sozialistische Republik", proklamiert wurde: Die Rote Armee war in Georgien einmarschiert und hatte die knapp dreijährige Unabhängigkeit des Landes beendet.

Die Verfassung von 1921 galt zur damaligen Zeit als eine der fortschrittlichsten und beinhaltete viele sozialdemokratische Elemente. Sie enthielt beispielsweise umfangreiche sozial-ökonomische Rechte (Kapitel XIII) und ein Kapitel mit Rechten nationaler Minderheiten (Kapitel XVI). Die staatsorganisationsrechtlichen Passagen in der Verfassung von 1921 waren dagegen kurz gehalten. Sie entwarfen eine parlamentarische Republik: Das Parlament konnte nicht aufgelöst werden. Es wählte den Vorsitzenden der Regierung auf ein Jahr. Eine Wiederwahl war nur einmal zulässig (Art. 67 GV von 1921). Der Regierungschef ernannte sodann sein Kabinett, ohne auf eine Zustimmung des Parlamentes angewiesen zu sein (Art. 68 GV von 1921). 


\title{
The Protection of Individual Rights in the Constitution of Colombia
}

\author{
By Andreas Timmermann
}

The starting point of this contribution is that the Colombian constitution reflects both the social and economical requests of a developing country and the most important principles of European constitutional law. The resulting compromise leads to a characteristic systematization and differentiation of individual rights. As a consequence the constitutional court handles the protection of individual rights rather flexibly. The court develops special guiding principles and accommodates European doctrines of fundamental rights to the social reality in Colombia.

\section{New Constitutional Structures in Georgia}

\section{By Wolfgang Gaul}

Over the last decade, the Republic of Georgia has been at the forefront of political changes within the Community of Independent States (CIS). What type of governmental structure should a country choose that has undergone such severe political changes? It needs to find a way to comfort the people's desire for security and stabilisation but should also avoid establishing authoritarian structures.

The current Georgian Constitution establishes a notable balance between President and Parliament. However, the vague responsibilities of the State Minister and the National Security Council tip the scale towards presidential powers. The article reveals which different approaches had been proposed during the constitution-making process and which constitutional archetypes influenced the key players of the country.

Transcultural legal consultation supported and escorted the different existing drafts. A compromise was elaborated during an international conference in Chicago. However, it all ended differently, when Shevardnadze and his Minister of Justice, Ninidze, turned the tide towards stronger presidential powers.

Will these powers be sufficient to unify a country which is on the verge of separation primarily due to ethnic differences or instead, should its regions and local authorities be granted more autonomy? The rules of the game are all laid out in the constitution - and the game itself is called politics. 